\title{
Influence of surface stress on frequency of microcantilever-based biosensors
}

\author{
Q. Ren, Y.-P. Zhao
}

Microsystem Technologies 10 (2004) 307-314

DOI: $10.1007 / s 00542-003-0329-4$

There is a print error in equation (21) as follows. It should be the 1st derivatives in the bracket, not 2 nd derivative.

WRONG form:

$E I \frac{\partial^{4} w}{\partial x^{4}}-\frac{\partial}{\partial x}\left(N_{(x)} \frac{\partial^{2} w}{\partial x^{2}}\right)+\rho A \frac{\partial^{2} w}{\partial t^{2}}=0$,

The CORRECT form:

$E I \frac{\partial^{4} w}{\partial x^{4}}-\frac{\partial}{\partial x}\left(N_{(x)} \frac{\partial w}{\partial x}\right)+\rho A \frac{\partial^{2} w}{\partial t^{2}}=0$, 